\title{
$P$-POINTS IN RANDOM UNIVERSES
}

\author{
PAUL E. COHEN
}

\begin{abstract}
A pathway is defined as an increasing sequence of subsets of ${ }^{\omega} \omega$ which satisfy certain closure and boundedness properties. The existence of a pathway is shown to imply the existence of a $P$-point in $\beta N \backslash N$. Pathways are shown to exist in any random extension of a model of $\mathrm{ZFC}+\mathrm{CH}$.
\end{abstract}

A point in a topological space is called a $P$-point if the intersection of any countable family of its neighborhood is a neighborhood. Shelah [8] has recently shown the existence of a $P$-point in $\beta N \backslash N$ to be independent of ZFC.

Various assumptions are known to imply the existence of $P$-points [1], [4], [6]. In this paper we contribute a new axiom of this sort and we show that the axiom holds in any random extension of a model of $\mathrm{ZFC}+\mathrm{CH}$. The referee has pointed out that Kunen has shown the existence of $P$-points in certain models of this description [5].

1. From pathways to $P$-points. If $f, g \in{ }^{\omega} \omega$ then $f \leqslant g$ is taken to mean that $\{n \mid f(n)>g(n)\}$ is finite. It will be convenient to identify a subset of $\omega$ with its characteristic function. Thus if $a, b \subseteq \omega$ then $a \leqslant b$ means that $a-b$ is finite.

An unbounded $f \in{ }^{\omega} \omega$ can be interpreted as a sequence

$$
\omega=f^{(0)} \supseteq f^{(1)} \supseteq \cdots
$$

where $f^{(n)}=\{k \in \omega \mid f(k)<n\}$. A free ultrafilter $U$ on $\omega$ is called a $P$-point provided that whenever $f \in{ }^{\omega} \omega$ is unbounded and $f^{(n)} \in U$ for all $n \in \omega$ there is an $a \in U$ such that $a \leqslant f^{(n)}$ for all $n \in \omega$. A discussion of the relationship of this definition to $\beta N$ can be found in [7].

Let $\kappa$ be the smallest cardinal of a $\leqslant$-dominating subset of ${ }^{\omega} \omega$. Ketonen [4] has shown that if $\kappa=2^{\omega}$ then there is a $P$-point. What follows is a refinement of his construction.

Call a sequence $\left\langle A_{\alpha} \mid \alpha<\kappa\right\rangle$ a pathway provided:

(a) $\cup_{\alpha<\kappa} A_{\alpha}={ }^{\omega} \omega$,

(b) $A_{\alpha} \subseteq A_{\beta}$ whenever $\alpha<\beta$,

(c) $A_{\alpha}$ does not $\leqslant$-dominate $A_{\alpha+1}$,

(d) $(f$ join $g) \in A_{\alpha}$ whenever $f, g \in A_{\alpha}$ (where $\left(f\right.$ join $g$ ) $=h \in{ }^{\omega} \omega$ is defined for $n \in \omega$ by $h(2 n)=f(n)$ and $h(2 n+1)=g(n))$,

Received by the editors May 19, 1978.

AMS (MOS) subject classifications (1970). Primary 04A30, 54D35.

$K e y$ words and phrases. Stone-Cech compactification, $P$-points, random forcing.

() 1979 American Mathematical Society 0002-9939/79/0000-0223/\$02.00 
(e) if $f \leqslant_{T} g$ and $g \in A_{\alpha}$ then $f \in A_{\alpha}$. The symbol $\leqslant_{T}$ is used for Turing reducibility.

1.1 TheOREM. The existence of a pathway implies the existence of a P-point.

Proof. We begin by listing some easily derived properties of the pathway $\left\langle A_{\alpha} \mid \alpha<\kappa\right\rangle$.

(i) $a \cap b \in A_{\alpha}$ whenever $a, b \in A_{\alpha}$,

(ii) if $f \in A_{\alpha}$ is unbounded then there is a $g \in A_{\alpha}$ where for all $n \in \omega, f^{(n)}$ has at least $n$ members smaller then $g(n)$,

(iii) if $f, g \in A_{\alpha}$ and $f$ is unbounded then $\left({ }_{g}^{f}\right) \in A_{\alpha}$ where

$$
\left(\begin{array}{l}
f \\
g
\end{array}\right)=\{k \in \omega \mid \exists n<f(k)[k<g(n)]\}
$$

(iv) if $f, g \in A_{\alpha}$ then $h \in A_{\alpha}$ where for $n \in \omega, h(n)=\min (f(n), g(n))$,

(v) if $f, g \in A_{\alpha}$ and $g \in{ }^{\omega} 2$ then $h \in A_{\alpha}$ where $h(n)=f(n) \cdot g(n)$.

Let us say that $U \subseteq{ }^{\omega} 2$ is a free filterbase if whenever $a, b \in U$ there is an infinite $c \in U$ with $c \subseteq a \cap b$.

By transfinite recursion we choose a sequence,

$$
U_{0} \subseteq U_{1} \subseteq \cdots \subseteq U_{\alpha} \subseteq \cdots \quad(\alpha<\kappa)
$$

where $U_{\alpha}$ is maximal among the free filterbases $X \subseteq A_{\alpha} \cap{ }^{\omega} 2$. If $U_{\alpha}$ has been chosen then by $c$ there is an $f_{\alpha} \in A_{\alpha+1}$ such that for no $g \in A_{\alpha}$ is $f \leqslant g$. Provided that

$$
U_{\alpha}^{*}=U_{\alpha} \cup\left\{\left(\begin{array}{l}
g \\
f_{\alpha}
\end{array}\right) \mid g \in A_{\alpha} \text { is unbounded } \wedge \forall n g^{(n)} \in U_{\alpha}\right\}
$$

is a free filterbase we require $U_{\alpha+1} \supseteq U_{\alpha}^{*}$. Notice that (iii) guarantees that $U_{\alpha}^{*} \subseteq A_{\alpha+1}$. We will show that $U=\cup_{\alpha<\kappa} U_{\alpha}$ is a $P$-point.

It must first be verified that each $U_{\alpha}^{*}$ is indeed a free filterbase; this will follow from I, II and III.

I. If $\left(\begin{array}{l}g \\ f_{\alpha}\end{array}\right) \in U_{\alpha}^{*}$, where $\forall n g^{(n)} \in U_{\alpha}$ then by (ii) there is an $h \in A_{\alpha}$ such that $g^{(n)}$ always has more than $n$ members smaller than $h(n)$. By the choice of $f_{\alpha}$ we have $f_{\alpha} \nless h$ and so $c=\left\{n \mid f_{\alpha}(n)>h(n)\right\}$ is infinite. But then

$$
\left(\begin{array}{l}
g \\
f_{\alpha}
\end{array}\right)=\bigcup_{n \in \omega}\left\{k<f_{\alpha}(n) \mid k \in g^{(n)}\right\}
$$

must be infinite since whenever $n \in c$

$$
\left\{k<f_{\alpha}(n) \mid k \in g^{(n)}\right\} \supseteq\left\{k<h(n) \mid k \in g^{(n)}\right\}
$$

has at least $n$ members.

II. Suppose $g_{1}, g_{2} \in A_{\alpha}$ are unbounded and $\forall n g_{1}^{(n)} \cap g_{2}^{(n)} \in U_{\alpha}$. By (iv), $g \in A_{\alpha}$ where

$$
g(n)=\min \left(g_{1}(n), g_{2}(n)\right)
$$

For $n \in \omega, g^{(n)}=g_{1}^{(n)} \cap g_{2}^{(n)}$. It follows that $\left(\begin{array}{l}g \\ f_{\alpha}\end{array}\right) \in U_{\alpha} \in U_{\alpha}^{*}$ and that

$$
\left(\begin{array}{c}
g \\
f_{\alpha}
\end{array}\right)=\left\{k \in \omega \mid \exists n<g(k)\left[k<f_{\alpha}(n)\right]\right\} \subseteq\left(\begin{array}{c}
g_{1} \\
f_{\alpha}
\end{array}\right) \cap\left(\begin{array}{c}
g_{2} \\
f_{\alpha}
\end{array}\right) \text {. }
$$


III. Suppose $a, g \in A_{\alpha}, a \in{ }^{\omega} 2, g$ is unbounded and $\forall n g^{(n)} \cap a \in A_{\alpha}$. By (v), $h \in A_{\alpha}$ where $h(n)=f(n) \cdot g(n)$. For $n \in \omega, h^{(n)}=g^{(n)} \cap a$. Thus

$$
\left(\begin{array}{l}
g \\
f_{\alpha}
\end{array}\right) \cap a=\left(\begin{array}{l}
h \\
f_{\alpha}
\end{array}\right) \in U_{\alpha}^{*}
$$

Since each $U_{\alpha}$ is a free filterbase so is $U$. If $a \subseteq \omega$ then for some $\alpha a \in A_{\alpha}$. Since either $a \in U_{\alpha}$ or $\omega-a \in U_{\alpha}$ we have that $U$ is an ultrafilter.

Suppose $g \in{ }^{\omega} \omega$ is unbounded and such that $\forall n \in \omega g^{(n)} \in U$. For some $\alpha, g \in A_{\alpha}$ and so $\forall n g^{(n)} \in U_{\alpha}$. But then $\left(\begin{array}{l}g \\ f_{\alpha}\end{array}\right) \in U_{\alpha+1} \subseteq U$ and for all $n \in \omega$, $\left(\begin{array}{l}g \\ f_{\mathrm{a}}\end{array}\right) \subseteq g^{(n)}$. Thus $U$ is a $P$-point.

2. Pathways in random universes. Let $\Re$ be a countable standard transitive model of ZFC in which $\lambda$ is an infinite ordinal number. Let $R=R_{\lambda}$ be the cartesian product in $\mathfrak{N}$ of $\lambda$ copies of 2 endowed with the product measure. In $\Re$, let $B=B_{\lambda}$ be the Boolean algebra of measurable sets modulo the sets of measure zero. Since $B$ is c.c.c. and countably complete it is complete in M.

If $H$ is $B$-generic over $\mathscr{N}$ and $r \in{ }^{\lambda} 2$ is such that for $\alpha<\lambda, r(\alpha)=1$ iff $\{f \in R \mid f(\alpha)=1\}$ is in a member of $H$, then $r$ is called random over $\mathfrak{N}$.

2.1 LEMMA. If $G$ is $B$-generic over $\mathfrak{R}$ then ${ }^{\omega} \omega \cap \mathfrak{T}$ dominates ${ }^{\omega} \omega \cap$ TL $[G]$.

Lemma 2.1 is proven in [10]. The development of Lemmas 2.2, 2.3 and 2.4 can be quite similar to that found in [9] (special care must be taken with the absoluteness arguments when $\lambda$ is uncountable). At the suggestion of the referee, the proofs of these lemmas are left as exercises for the reader.

2.2 LEMMA. Suppose $\mathfrak{R} \supseteq \mathfrak{T}$ where $\mathfrak{T}$ is a countable standard transitive model of ZFC. If $r \in{ }^{\lambda} 2$ is random over $\Re$ then it is also random over $\Re$.

2.3 LeMma. If $r \in{ }^{\lambda} 2$ is random over $\mathfrak{N}$ and $\Gamma \in\left({ }^{\omega} \lambda \cap \mathfrak{T}\right)$ is injective then $r \circ \Gamma \in{ }^{\omega} 2$ is random over $\mathfrak{M}$.

2.4 LEMma. Suppose $\Re$ is a model of ZFC such that for each $s \in{ }^{\omega} 2 \cap$ $\mathbb{R}$ there is an $\alpha<\omega_{1}$ such that $L_{\alpha}[s]$ is a model of ZFC. If $r \in{ }^{\lambda} 2$ is random over $\mathfrak{M}$ then for every $t \in{ }^{\omega} 2 \cap \mathfrak{M}[r]$ there is an $\alpha<\omega_{1}$, an $s \in{ }^{\omega} 2 \cap \mathfrak{M}$ and an injective $\Gamma \in{ }^{\omega} \lambda \cap \mathfrak{N}$ such that $t \in L_{\alpha}[r \circ \Gamma, s]$.

2.5 THEOREM. If $\Re$ is a model of $Z F C+C H, \nu$ is an infinite ordinal of $\Re$ and $r \in{ }^{\nu} 2$ is random over $\mathfrak{N}$ then there is a pathway in $\mathfrak{M}[r]$. By Theorem 1.1 it follows that there is a P-point in $\mathscr{M}[r]$.

Proof. We first observe that we may weaken clause $\mathrm{c}$ in the definition of a pathway to the statement that " $A_{\alpha}$ does not dominate ${ }^{\omega} \omega$. ."

In Lemma 2.4 it was assumed that the $L_{\alpha}[s]$ were models of ZFC. For the result to hold, however, it is necessary only that the $L_{\alpha}[s]$ be models of some finite fragment $\Phi_{1}$ of ZFC. Similarly, by Lemma 2.1 there is a finite fragment $\Phi \supseteq \Phi_{1}$ of ZFC such that whenever $L_{\alpha}[s]$ is a model of $\Phi$ and $r \in{ }^{\omega} 2$ is 
random over $L_{\alpha}[s]$ then ${ }^{\omega} \omega \cap L_{\alpha}[s] \leqslant-$ dominates ${ }^{\omega} \omega \cap L_{\alpha}[r, s]$. For each $s \in{ }^{\omega} 2 \cap \Re$, we know by the reflection principle that there are arbitrarily large $\alpha<\omega_{1}$ for which $L_{\alpha}[s]$ is a model of $\Phi$.

In $\Re$, choose a sequence $\left\langle s_{\alpha} \mid \alpha<\omega_{1}\right\rangle$ from ${ }^{\omega} 2$ and an increasing sequence $\left\langle\xi_{\alpha} \mid \alpha<\omega_{1}\right\rangle$ from $\omega_{1}$ such that if $\mathfrak{N}_{\alpha}$ is defined as $L_{\xi_{\alpha}}\left[s_{\alpha}\right]$ then

A. $\mathfrak{\pi}_{\alpha}$ is a model of $\Phi$,

B. $\alpha<\beta<\omega_{1}$ implies $\mathfrak{N}_{\alpha} \subseteq \mathfrak{N}_{\beta}$,

C. ${ }^{\omega} 2 \cap \mathfrak{R}=\cup_{\alpha<\omega_{1}}\left({ }^{\omega} 2 \cap \mathfrak{T}_{\alpha}\right)$.

Let $\&$ be the set of all injective $\Gamma \in \omega_{\nu} \cap \Re$ and for $\alpha<\omega_{1}$ define

$$
A_{\alpha}=\bigcup\left\{{ }^{\omega} \omega \cap \Re_{\alpha}[r \circ \Gamma] \mid \Gamma \in \mathbb{S}\right\} .
$$

It is easy to see that $A_{\alpha}$ is closed under Turing reductions and finite joints. Since the (in $\mathscr{N}[r]$ ) countable set ${ }^{\omega} \omega \cap \Re_{\alpha}<$-dominates $A_{\alpha}$ it is clear that $A_{\alpha}$ cannot dominate ${ }^{\omega} \omega \cap \mathfrak{N}[r]$. Finally, since by 2.4 ,

$$
\bigcup_{\alpha<\omega_{1}} A_{\alpha}={ }^{\omega} \omega \cap \mathfrak{M}[r]
$$

it follows that $\left\langle A_{\alpha}\right| \alpha\left\langle\omega_{1}^{[r]}\right\rangle$ is a pathway in $\Re[r]$.

\section{REFERENCES}

1. A. Blass, The Rudin-Keisler ordering of P-points, Trans. Amer. Math. Soc. 179 (1973), $145-166$.

2. P. Halmos, Measure theory, Van Nostrand, New York, 1950.

3. T. Jech, Lectures in set theory with particular emphasis on the method of forcing, Lecture Notes in Math., Vol. 217, Springer, Berlin, 1971.

4. J. Ketonen, On the existence of P-points in the Stone-Cech compactification of the integers, Fund. Math. 42 (1976), 91-94.

5. K. Kunen, $P$-points in random real extensions (unpublished note).

6. A. Mathias, Happy families (to appear).

7. M. Rudin, Lectures on set theoretic topology, CBMS Regional Conf. Ser. in Math., no. 23, Amer. Math. Soc., Providence, R. I., 1975.

8. S. Shelah, On P-points, $\beta(\omega)$ and other results in general topology, Notices Amer. Math. Soc. 25 (1978), A-365, Abstract \#87T-G49.

9. R. Solovay, A model of set-theory in which every set of reals is Lebesgue measurable, Ann. of Math. (2) 92 (1970), 1-56.

10. G. Takeuti and W. Zaring, Axiomatic set theory, Springer, Berlin, 1973.

Department of Mathematics, Lehigh University, Bethlehem, Pennsylvania 18015 